\title{
Scanning tunneling spectroscopy of quantum well and surface states of thin Ag films grown on $\operatorname{GaAs}(110)$
}

\author{
C.-S. Jiang, ${ }^{1,2}$ H.-B. Yu, ${ }^{1}$ X.-D. Wang, ${ }^{1}$ C.-K. Shih, ${ }^{1}$ and Ph. Ebert ${ }^{1,3}$ \\ ${ }^{1}$ Department of Physics, University of Texas, Austin, Texas 78712 \\ ${ }^{2}$ Department of Physics, University of Tennessee, Knoxville, Tennessee 37996 \\ ${ }^{3}$ Institut für Festkörperforschung, Forschungszentrum Jülich GmbH, 52425 Jülich, Germany \\ (Received 31 October 2000; revised manuscript received 25 April 2001; published 19 November 2001)
}

\begin{abstract}
We investigate the electronic states of thin Ag films grown on $\operatorname{GaAs}(110)$ surfaces at low temperatures by scanning tunneling spectroscopy with single-layer thickness resolution. We identify the quantum-well states arising from the $z$ confinement of the two-dimensional Ag films, and find an unoccupied Shockley-type surface state $180 \pm 30 \mathrm{meV}$ above the Fermi energy. The $s-p$ electronic band dispersion along the $\Gamma-L$ direction is found to be shifted upward by $190 \pm 20 \mathrm{meV}$ compared to pure $\operatorname{Ag}(111)$ surfaces. This shift, and the fact that the Shockley-type surface state is unoccupied and thus also shifted upward compared to pure $\mathrm{Ag}(111)$ surfaces are connected to the lattice strain of the quasiperiodically modulated Ag film. Implications of the results for other $\mathrm{Ag}$ thin films are discussed.
\end{abstract}

DOI: 10.1103/PhysRevB.64.235410

PACS number(s): 73.20.-r, 73.61.- r, 68.55.-a

The growth of atomically flat two-dimensional metal overlayers on semiconductor substrates at low temperatures, followed by room-temperature annealing, ${ }^{1}$ attracted considerable attention recently, because of the unusual growth mode involved and the possibility to achieve atomically flat films for nonwetting systems. The exceptional growth morphology has been related to the electronic structure of the two-dimensional metal film, and a so-called "electronic growth mechanism" has been postulated. ${ }^{2}$ Thus a direct correlation of the growth morphology with the electronic properties of the thin films is a key to the understanding of underlying physics. Although Ag films were so far grown on a number of substrates, the films were either investigated structurally $^{1,3-6}$ or in view of their electronic properties. ${ }^{7-9}$ However, no work simultaneously measured the surface structure and the electronic properties. Such an investigation is even more crucial in the light that very subtle changes in the growth conditions can flip the growth mode from two to three dimensional, thus making the interpretation of macroscopic techniques, such as photoelectron spectroscopy difficult.

In the conventional picture, thin Ag films exhibit a twodimensional confinement of electrons giving rise to quantum-well states $^{2,7-9}$ and a Shockley-type surface state trapped in a potential well arising from a gap in the surfaceprojected bulk band structure and the potential barrier at the metal-vacuum interface. ${ }^{9-13}$ However, the surface state itself has not been observed so far for thin $\mathrm{Ag}(111)$ films grown on GaAs(110) and $\mathrm{Si}(111)$ substrates. ${ }^{7,9}$ This was tentatively explained by a shift of the surface state above the Fermi energy $\left(E_{F}\right)$ making it inaccessible with photoelectron spectroscopy. ${ }^{9}$ However, a direct proof is still lacking, and nothing is known about the energy position of the possible surface state. In addition, such a shift should affect the other electronic properties too, but nothing has been found so far questioning the above explanation.

In view of the still unclear electronic properties and the missing simultaneous structural and electronic investigation, in the present paper we investigate the electronic structure of thin atomically flat $\mathrm{Ag}$ films grown on $\mathrm{GaAs}(110)$ substrates as a function of the layer thickness of the Ag films using spatially resolved scanning tunneling spectroscopy (STS). Spatially resolved scanning tunneling spectroscopy allows us to probe both occupied and empty states, as well as real space and electronic properties simultaneously. We identify the individual quantum-well states and the existence of a Shockley-type surface state, and extract the dispersion of the $s-p$ electronic band. We show that the Shockley-type surface state and the $s$ - $p$ band close to the $L$ point are both shifted upward compared to pure $\operatorname{Ag}(111)$ surfaces. We connect these effects with the lattice strain present in a quasiperiodically modulated thin Ag film on GaAs. Furthermore, we provide additional support for the identification of the surface state by selectively controlling the surface states' shifts by evaporation of potassium $(\mathrm{K})$ on the $\mathrm{Ag}$ surface. Our results allow us to provide a comprehensive picture of the electronic properties with a direct correlation to the atomic scale surface morphology.

The atomically flat $\mathrm{Ag}$ films were grown on $\mathrm{GaAs}(110)$ surfaces cleaved in ultrahigh vacuum using a two-step growth procedure. ${ }^{1}$ The Ag was first deposited at liquid-He temperature followed by a warm up to room temperature within 3 to $12 \mathrm{~h}$. This procedure yields flat two-dimensional Ag films with a (111) orientation ${ }^{4}$ and a quasiperiodic superstructure. ${ }^{5}$ Figure 1(a) shows a constant-current scanning tunneling microscope image of the quasiperiodic superstructure of the Ag film. The presence of the superstructure implies that the $\operatorname{Ag}(111)$ film is strained. Detailed low-energy electron-diffraction (LEED) patterns indicate that the [111] direction of the Ag film is not exactly aligned with the [110] direction of the GaAs substrate. Instead, it is tilted by about $4^{\circ}$ (Refs. 3 and 4 ) toward the GaAs [001] axis. The importance of the finding of the superstructure will be shown below.

On Ag films, such as the one shown in Fig. 1(a) we measured current-voltage spectra and deduced the quantity $(d I / d V) /(\overline{I / V})$, which is proportional to the density of states. ${ }^{14}$ Figure 1(b) shows the STS spectra obtained on a variety of Ag films with thickness ranging between 2.0 and 
(a) LSLLSLLSLSLLSLSLLSLSLLSLSLLSLSL

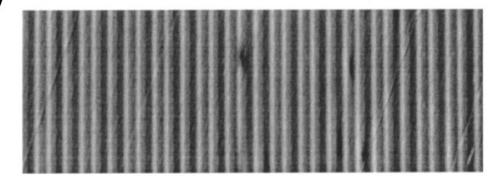

(b)

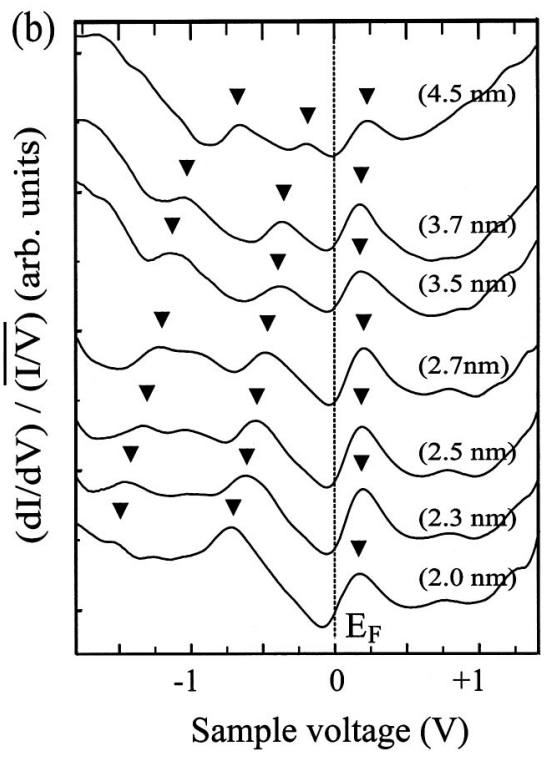

FIG. 1. (a) Constant-current scanning tunneling microscopy image of a Ag film grown on $\mathrm{GaAs}(110)$. The (111)-oriented surface of the film is quasiperiodically modulated (corrugation $\sim 0.05 \mathrm{~nm}$ ), and consists of long and short segments as indicated in the figure $(L$ and $S$ ). (b) Scanning tunneling microscope spectra of the Ag films with thicknesses of $2.0-4.5 \mathrm{~nm}$. The tip-sample separation has been fixed by a sample voltage of $-0.5 \mathrm{~V}$ and a tunnel current of $0.5 \mathrm{nA}$. Each spectra is an average of 10000-40000 individual spectra in order to reduce the signal to noise ratio. The spectra are displayed as $(d I / d V) /(\overline{I / V})$ (Ref. 14), which is proportional to the local density of state.

$4.5 \mathrm{~nm}$. Note that we determined the thickness of the film on which we measured the particular spectra by measuring spectra not only on a variety of different $\mathrm{Ag}$ films with varying thicknesses but also by taking advantage of the real-space resolution, and acquiring spectra on individual terraces separated by one atomic layer high steps. In this way we directly correlate the tunneling spectra and the film thickness. Furthermore, our technique automatically assures that we obtained data for flat films and not three-dimensional mounts frequently found due to inappropriate film preparation conditions.

Each spectrum in Fig. 1(b) is the an average of $10000-$ 40000 individual spectra in order to enhance the signal-tonoise ratio. All spectra exhibit three prominent peaks in the density of states. Two of them are occupied states (negative sample voltages), while one is an unoccupied state (positive sample voltage). The energy positions of the occupied states [peaks 1 and 2 in Fig. 2(a)] change with increasing film thickness, whereas the unoccupied state [peak 3 in Fig. 2(a)] does not change much in energy. The occupied states can be correlated with the quantum-well states arising from the $z$ confinement of the thin Ag film, whereas the empty state is a (a)

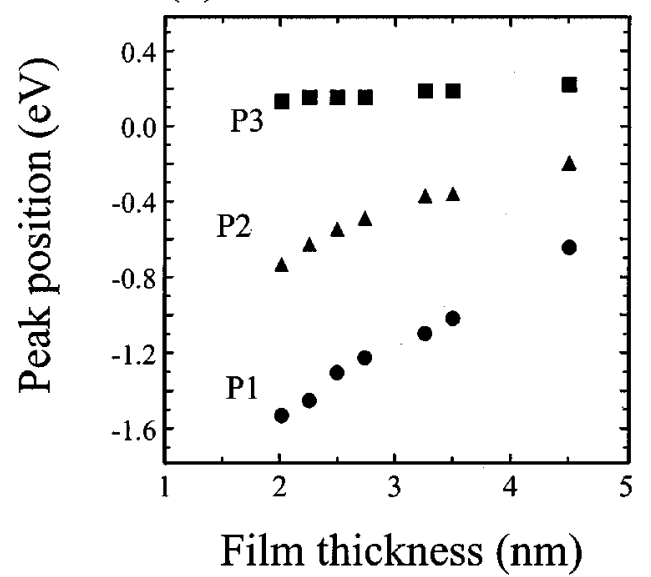

(b)

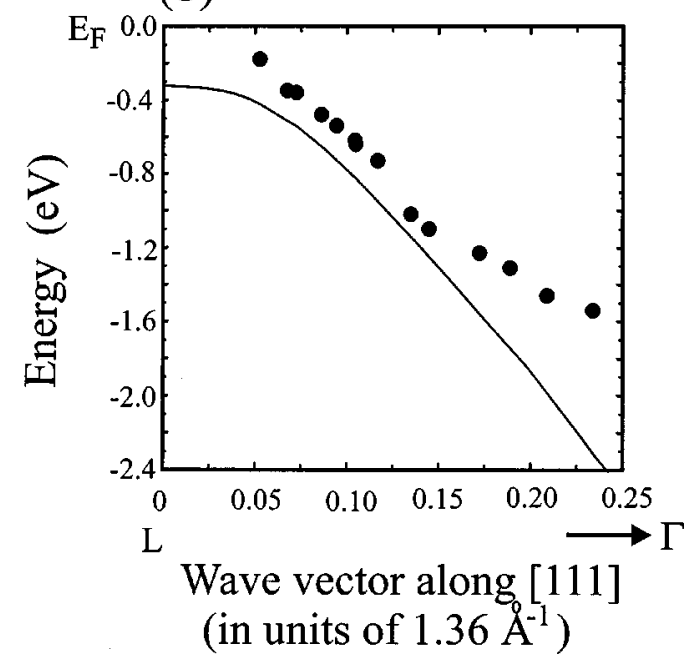

FIG. 2. (a) Energy locations of the peaks in the scanning tunneling microscope spectra in Fig. 1(b) as a function of the film thickness. $P 1$ and $P 2$ are occupied states, whereas $P 3$ is an unoccupied state. (b) $s$ - $p$ band structure of the Ag film around the $L$ point along the $\Gamma-L$ direction. Circles show the measured data deduced from the energy positions of the quantum well states in the spectra of Fig. 1(b) (see the text). The solid line represents a theoretical calculation of the band structure for the bulk fcc Ag along the $\Gamma-L$ direction (Ref. 15).

Shockley-type surface state, as shown below. Note that we took exceptional care to avoid tip-induced artifacts in the spectra by acquiring a very large data base. In the present work, we focus only on the reproducible features in the spectra.

For the interpretation of the spectra, we first recall the electronic properties of pure Ag. Figure 2(b) shows the part of the band structure of pure Ag relevant for our experiments as the solid line. It is an $s-p$ band. ${ }^{15}$ The $s-p$ band levels off the Fermi energy $E_{F}$ at the $L$ point on the boundary of the first Brillouin zone, and decreases monotonically in energy toward the $\Gamma$ point. There is a band gap around the $L$ point. The Fermi level is positioned in this energy gap above the $L$ point.

In a thin Ag film the wave functions of the electrons are confined due to the potential barriers at the Ag-vacuum and 
the Ag-GaAs interfaces. The wave vector of the stationary quantum well states is given by

$$
k_{z}=(n \pi / N a)+C
$$

where $a$ is the atomic layer distance, $N$ is the number of atomic layers of the metal film, and $n$ is the quantum number. The correction $C$ comes from the phase shifts at both the $\mathrm{Ag}$-vacuum and Ag-GaAs interfaces due to the finite height of the potential barriers forming the well. Equation (1) shows that the energetic position of the quantum-well states in the $s-p$ band and their $k$ values depend strongly on the film thickness.

The observation of a shift in energy of the occupied peaks in the spectra shown in Fig. 1(b), with increasing thickness of the Ag films, can be taken as an indication that the peaks are signatures of quantum-well states in the two-dimensional Ag film. This assignment of the occupied peaks to quantumwell states allows us to extract further information. Combining the measured energy positions of the quantum-well states and the film thickness, it is possible to derive the dispersion of the $s-p$ band in the thin Ag film. ${ }^{8,16}$ For this purpose one has to recall that the quantum number $n$ in the first Brillouin zone should be between 1 and $N$. Thus the first occupied state below the $E_{F}$ (peak 2) corresponds to a quantum-well state with the quantum number $n=N-1$, and the wave vector is $k_{z}=(N-1) \pi / N a$. If we measure the wave vector from the boundary of the Brillouin zone ( $L$ point), the wave vector of the state is $k_{z L}=\pi / a-k_{z}=\pi / N a$. In analogy, the wave vector of the neighboring state (peak 1) visible in the same STS spectrum in Fig. 1(b) is $k_{z L}=2 \pi / N a$. By measuring spectra on Ag films with different thicknesses, we effectively vary the wave vectors, and thus obtain the dispersion from the energy locations of the quantum well. Figure 2(b) shows the obtained band structure along the $\Gamma-L$ direction. Note that we assumed that the correction $C$ arising from the finite energy barriers can be neglected, as discussed below. Close to the $L$ point, the measured data have a similar shape as compared to the theoretical dispersion for pure $\operatorname{Ag}(111)$ shown by the solid line. The experimentally obtained energy values of the thin Ag film are, however, shifted upward by 190 $\pm 20 \mathrm{meV}$ relative to the theoretical values for pure $\mathrm{Ag}(111)$. With increasing distance from the $L$ point, the experimental data points deviate increasingly from the theoretical dispersion toward higher energies. Thus the dispersion and the bandwidth of $\mathrm{Ag}$ thin films on $\mathrm{GaAs}(110)$ substrates are smaller than those of the theoretical band structure of pure $\operatorname{Ag}(111)$.

At this stage we briefly discuss the effect of the interfaces. The interfaces affect the correction $C$ in Eq. (1). Although the $k$ values sensitively depend on the correction $C$, such that the overall band shape would be shifted horizontally, it does not affect our main conclusion that the band in the region close to the $L$ point is shifted upward, because the measured energy location of the band at the smallest $k$ value [see Fig. $2(b)]$ is higher in energy than the maximum energy of the $s-p$ band of pure $\operatorname{Ag}(111)$ [the solid line in Fig. 2(b)]. It seems that the correction $C$ can be reasonably neglected in the range of energies and film thickness of interests here, ${ }^{7,9,17}$

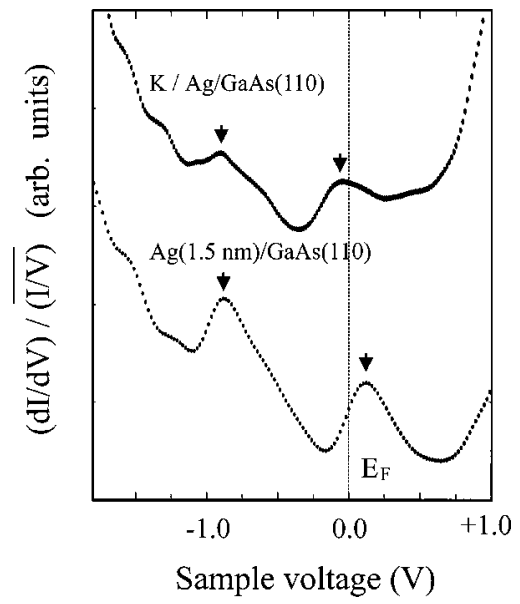

FIG. 3. Scanning tunneling microscope spectra taken on the clean surface of a 1.5-nm thick thin Ag film on GaAs(110) and after deposition of $0.2 \mathrm{~nm}$ potassium on top of the Ag film. The unoccupied state, which is shown to be the Shockley-type surface state, shifts about $190 \mathrm{meV}$ downward, and is filled due to the lowering of the work function by the deposition of potassium.

because, on the one hand, the phase shift at the Ag-vacuum interface varies only little with changing film thickness, and its effect is minor. ${ }^{9,18,19}$ On the other hand, $E_{F}$ lies at the $\mathrm{Ag}-\mathrm{GaAs}$ interface within the GaAs band gap. Furthermore, GaAs(110) surfaces have no surface states in the gap. Thus electrons with energies close to $E_{F}$ are Bragg reflected at the $\mathrm{Ag} / \mathrm{GaAs}$ interface, and the phase shift is roughly $\pi$. From this we conclude that indeed the $s-p$ band of the thin film is shifted upward at the $L$ point compared to that of the bulk material. ${ }^{20}$ This physical origin of the shift will be discussed at a later stage, when all data are presented.

In addition to the quantum-well states, we also observed a peak at $\sim 180 \mathrm{meV}$ above $E_{F}$ which does not show any dependence on the film thickness. The lack of any thickness dependence of this feature indicates that it is a surfacerelated state not connected with the "bulk" properties of the thin film. On $\operatorname{Ag}(111)$ surfaces, the surface related feature is a Shockley-type surface state.

The identification of the surface state can be most easily corroborated by the deposition of, for example, potassium on the Ag surface, which most strongly affects the surface states and affects the bulk-related states far less. Figure 3 shows that after deposition of $\mathrm{K}$ only the energy of the empty state decreases by $\sim 190 \mathrm{meV}$, and the state is thus filled. The other states remain essentially unaffected. These effects of potassium can be understood by taking into account that potassium lowers the work function: In the framework of the phase-accumulation model, the Shockley state shifts toward a lower energy due to the decrease of the vacuum barrier, i.e., the work function. The $\sim 190 \mathrm{meV}$ shift of the Shockley state observed in the STS spectra would result from a decrease in the work function by $1.2 \mathrm{eV}$. ${ }^{9}$ Similar shifts of the Shockley state, obtained by lowering the work functions, were also observed on Ag films grown on $\mathrm{Si}(111)$ surfaces ${ }^{9}$ and on the surfaces of other noble metals. ${ }^{24,25}$ This experiment therefore provides a further support for the interpreta- 
tion that the maximum observed in the empty states in the STS spectra is indeed a Shockley-type surface state.

Note that the energy of the quantum-well state in Fig. 3 does not change with the deposition of potassium, although the lowering of the work function should increase the extension of the wave-function tails of the quantum-well states into the vacuum barrier. In other words, it would change the phase shift on the potential wall at the metal-vacuum interface, and thus the wave vector of the standing waves. However, in a rough estimation, a decrease in the work function of about $1.2 \mathrm{eV}$ results in a change of the wave vector of the first quantum-well state of only $\Delta k_{Z(n=N-1)}<0.05 \mathrm{~nm}^{-1}$, which corresponds to a shift in the energy of the quantumwell state of less than $20 \mathrm{meV}$. Such a small shift cannot be resolved in our measurement at room temperature. This further supports the approximations used above in the extraction of the dispersion of the $s-p$ band.

After the identification of the surface state on $\mathrm{Ag} /$ $\operatorname{GaAs}(110)$ it is important to address the energy position of the surface state. We recall that on a pure (and unstrained) $\mathrm{Ag}(111)$ surface the surface state is about $30 \mathrm{meV}$ below $E_{F}$, and consequently fully occupied. ${ }^{9}$ Here however, we observe a surface state $180 \pm 30 \mathrm{meV}$ above the Fermi energy and thus depopulated. From this we infer that in the particular system the surface state is shifted upward by 210 $\pm 30 \mathrm{meV}$. The shift is in size and direction comparable to the shift observed for the $s-p$ band dispersion. This direct analogy suggests a common reason.

The shift in energy of the $s-p$ band and the surface state as well as the considerably reduced bandwidth of the dispersion of the thin Ag film on $\operatorname{GaAs}(110)$ as compared to pure $\mathrm{Ag}(111)$ can be related to the lattice strain in the thin $\mathrm{Ag}$ film. It has been shown that with increasing temperature noble metal single crystals exhibit a shift of the band structure toward $E_{F},{ }^{12,26}$ due to the thermal lattice constant expansion at high temperatures. The lattice expansion causes a decrease in the overlapping of the wave function, reducing the bandwidth. Using the result of a calculation based on the empirical pseudopotential method, ${ }^{9}$ we estimate that a shift of about $200 \mathrm{meV}$ corresponds to an in-plane tensile strain $\Delta a \cong 1 \%$. We recall that the thin Ag film on GaAs(110) exhibits a superstructure. The presence of a superstructure suggests that the film is strained. We can corroborate the presence of strain by taking into account that the (111) orientation of the $\mathrm{Ag}$ film is inclined by about $4^{\circ}$ with respect to the [110] orientation of the GaAs substrate according to LEED studies. ${ }^{3,4}$ If we assume that the Ag-GaAs interface has the same inclination, we can describe the $\mathrm{Ag}$ film as a (667)-oriented film having (111) microfacets. In such a structure the distance between two [1 $1 \overline{1} 0]$ steps on the vicinal $\mathrm{Ag}(667)$ surface is $3.16 \mathrm{~nm}$, which is roughly two times the average separation $(1.5 \mathrm{~nm})$ of the stripes on the quasiperiodically modulated surface. ${ }^{5}$ In this configuration the unrelaxed $\operatorname{Ag}(667)$ film should have a large lattice mismatch of about $6 \%$ compared to the GaAs(110) lattice along the [001] direction. It is conceivable that this lattice mismatch is partially relaxed by the quasiperiodic superstructure, leading to an internal lattice strain. Note that we observed identical quasiperiodic superstructures on all $\mathrm{Ag}$ films independent of the film thickness. From this we infer that the upward shift of the $s-p$ electronic band is induced by strain in the thin film.

The upward shift of the surface state can again be related to the upward shift of the $s-p$ electronic band and the effect of lattice strain: In the phase-accumulation model the Shockley state is the lowest eigenstate of the potential well, comprised of the image potential barrier on the vacuum side and the band gap of the crystal on the other side. The energy of the Shockley state thus depends on the height of the vacuum barrier, i.e., the work function, and also on the energy location of the band-gap edge (the $L$ point), which is shifted upward as we just discussed by $190 \pm 20 \mathrm{meV}$ compared to pure fcc Ag at the $L$ point. This upward shift leads then directly to an upward shift of the Shockley state from $\sim 30$ meV below the $E_{F}$ into the empty states above $E_{F}$.

Finally, one might wonder why no quantum-well states are found in the unoccupied density of states, despite the observation of an unoccupied surface state. This can be resolved as follows: The $s-p$ electronic band of fcc Ag has a wide band gap of $4.2 \mathrm{eV}$ along the $\Gamma-L$ direction above the $L$ point. ${ }^{15}$ The range of positive sample voltages $(0-1.5 \mathrm{~V})$ used in our study is within the band-gap energy. With increasing voltage, the electron energy would thus only increase along the lateral directions $(\Gamma-X$ and $\Gamma-W)$, while the energy along the $\Gamma-L$ direction must remain unchanged. Since quantum confinement occurs only along the direction normal to the film surface (the $\Gamma-L$ direction), there can be no quantum-well states in the empty states for the voltage range studied here. Higher voltages could not be applied to the film without destruction.

In conclusion, we probed, simultaneously with the topographic information, the electronic states of thin twodimensional Ag films grown on $\operatorname{GaAs}(110)$ using scanning tunneling spectroscopy as a function of the film thickness with single atomic layer resolution. In the spectra we directly identified occupied quantum-well states and one empty Shockley-type surface state. We deduced the $s-p$ band structure along the $\Gamma-L$ direction, and demonstrated that both the $s-p$ band and the Shockley surface state are shifted toward higher energies compared to the pure $\operatorname{Ag}(111)$ surface. This is explained by lattice strain in the thin $\mathrm{Ag}$ films on GaAs(110) surfaces.

The results deduced here can naturally be applied to $\mathrm{Ag}$ films grown on other substrates. In particular, it can be expected that in all strained films the surface states and the band structure will be shifted to higher energies. This happens in a number of cases where sometimes distinct superstructures can be found (e.g., GaAs and GaP). In those cases the surface state is depopulated, and can no longer be observed in photoelectron spectroscopy, which has mainly been used to characterize such films. Thus for a characterization of the energy shifts, scanning tunneling spectroscopy is the technique of choice.

This work was supported by NSF Grant Nos. DMR0071893 and DMR-9705406, NSF Science and Technology Center Grant No. CHE-8920120, and the Texas Advanced Research Program. 
${ }^{1}$ A. R. Smith, K.-J. Chao, Q. Niu, and C.-K. Shih, Science 273, 226 (1996).

${ }^{2}$ Z. Zhang, Q. Niu, and C. K. Shih, Phys. Rev. Lett. 80, 5381 (1998).

${ }^{3}$ G. Neuhold, L. Bartels, J. J. Paggel, and K. Horn, Surf. Sci. 376, 1 (1997).

${ }^{4}$ K.-J. Chao, Z. Zhang, Ph. Ebert, and C. K. Shih, Phys. Rev. B 60, 4988 (1999).

${ }^{5}$ Ph. Ebert, K.-J. Chao, Q. Niu, and C.-K. Shih, Phys. Rev. Lett. 83, 3222 (1999).

${ }^{6}$ M. M. R. Evans, B. Y. Han, and J. H. Weaver, Surf. Sci. 465, 90 (2000).

${ }^{7}$ D. A. Evans, M. Alonso, R. Cimino, and K. Horn, Phys. Rev. Lett. 70, 3483 (1993).

${ }^{8}$ J. J. Paggel, T. Miller, and T.-C. Chiang, Science 283, 1709 (1999)

${ }^{9}$ G. Neuhold and K. Horn, Phys. Rev. Lett. 78, 1327 (1997).

${ }^{10}$ W. Shockley, Phys. Rev. 56, 317 (1939).

${ }^{11}$ M.-C. Desjonqueres and D. Spanjaard, Concepts in Surface Physics (Springer-Verlag, Berlin, 1993), p. 182.

${ }^{12}$ R. Paniago, R. Matzdorf, G. Meister, and A. Goldmann, Surf. Sci. 336, 113 (1995).

${ }^{13}$ J. A. Knapp, F. J. Himpsel, A. R. Williams, and D. E. Eastman, Phys. Rev. B 19, 2844 (1979).

${ }^{14}$ R. M. Feenstra, J. Vac. Sci. Technol. B 7, 925 (1989); P. Martensson and R. M. Feenstra, Phys. Rev. B 39, 7744 (1989); C. K. Shih, R. M. Feenstra, and G. V. Chandrashekhar, ibid. 43, 7913 (1991).

${ }^{15}$ A.-B. Chen and B. Segall, Phys. Rev. B 12, 600 (1975).
${ }^{16}$ J. E. Ortega, F. J. Himpsel, G. J. Mankey, and R. F. Willis, Phys. Rev. B 47, 1540 (1993).

${ }^{17}$ Some photoemission studies (e.g., Refs. 8 and 16) obtained the quantities of the phase shift by fitting the photoemission data. The phase shift matches the data well at larger binding energy and thicker films than what we investigated in the present work, but poor agreement is found for films similar to ours. Moreover, their samples are metal films on metal substrates, which have significant phase shifts at the metal-metal interface increasing the total phase shift obtained by the fitting procedure.

${ }^{18}$ N. V. Smith, Phys. Rev. B 32, 3549 (1985).

${ }^{19}$ N. V. Smith, N. B. Brookes, Y. Chang, and P. D. Johnson, Phys. Rev. B 49, 332 (1994).

${ }^{20}$ Of course, this conclusion depends on the assignment of the $L_{4-}$ energy location for the pure $\operatorname{Ag}(111)$ surface. We noted that in the work of Ref. $21 L_{4-}$ was chosen to be only $80 \mathrm{meV}$ below $E_{F}$, different from the $0.3 \mathrm{eV}$ below $E_{F}$ used in our work. The majority of the work in the literature, however, refers to the $L_{4-}$ position as being $\sim 0.3 \mathrm{eV}$ below $E_{F}$, including many recent experiment measurements (see Refs. 9, 12, 15, 22, and 23).

${ }^{21}$ T. C. Hsieh, T. Miller, and T.-C. Chiang, Phys. Rev. Lett. 55, 2483 (1985).

${ }^{22}$ R. C. Jaklevic and J. Lambe, Phys. Rev. B 12, 4146 (1975).

${ }^{23}$ I. Matsuda, H. W. Yeom, T. Tanikawa, K. Tono, T. Nagao, S. Hasegawa, and T. Ohta, Phys. Rev. B 63, 125325 (2001).

${ }^{24}$ S. A. Lindgren and L. Walldén, Solid State Commun. 28, 283 (1978).

${ }^{25}$ S. A. Lindgren and L. Walldén, Solid State Commun. 34, 671 (1980).

${ }^{26}$ N. E. Christensen, Phys. Rev. B 20, 3205 (1979). 